\title{
A 70-Year-old Male With Abdominal Pain
}

\author{
William H Chong, MD
}

\section{Introduction}

Acute pancreatitisis an acuteinflammatory process of the pancreas. It is usually associated with severe acute upper abdominal pain and elevated blood levels of pancreatic enzymes. ${ }^{1}$ Serum amylase and lipase are common tests obtained as biochemical markers for acute pancreatitis. ${ }^{2}$ Pancreatitis, however, is not the sole cause of elevated pancreatic enzymes. Non-pancreatic causes of hyperamylasemia include inflammation or trauma to the salivary glands, bowel perforation or infarction, renal failure, abdominal trauma, and macroamylasemia. ${ }^{2}$ An unusual cause of elevated pancreatic enzymes is reported in this case report.

\section{Case Presentation}

A 70 year old male was admitted to the hospital after being evaluated by his primary care physician for abdominal pain. He initially complained of abdominal pain and had labs and $\mathrm{x}$-rays ordered from the office. After 2 days, he called for results and was admitted to the hospital for abnormal lab studies.

At admission, he described his pain as dull and achy, located primarily in the right upper quadrant. Initially the pain was rated a $7 / 10$ in severity, but had improved to $2 / 10$ by the time of admission. The only treatment he had used over this period was ibuprofen. His symptoms were worse with deep inspiration, and he noted no other symptoms such as nausea, vomiting, constipation, or diarrhea.

His past medical history was significant for benign prostatic hypertrophy, hyperlipidemia, coronary artery disease, reflux, and vitiligo. Past surgeries included appendectomy and hand surgery. He had no allergies and the only medication that he was taking was terazosin. Social history was negative for alcohol, tobacco, or drugs, and family history was non-contributory. Review of systems was otherwise unremarkable.

On physical exam he was in no acute distress and his vital signs were stable. Cardiovascular and pulmonary exam were unremarkable. Abdominal exam was significant for tenderness to palpation in the right upper quadrant over the rib cage, but a negative Murphy's sign. Labs and studies obtained from his physician's office were significant for a serum amylase level of 699 units/dl. Other labs including complete blood count, electrolytes, and lipase were all within normal limits. X-rays of his ribs and spine showed no evidence of fractures.

His physician admitted him with concern for pancreatitis. Further studies obtained while admitted to the hospital including abdominal ultrasound and CT scan showed no evidence of pancreatitis or gallstones. Serial amylase levels continued to be elevated throughout his hospitalization. His pain was attributed to chondritis from having moved furniture prior to initial presentation. An amylase-creatinine clearance ratio was calculated and found to be low at $0.2 \%$, consistent with macroamylasemia, and his elevated amylase level was attributed to macroamylasemia.

\section{Discussion}

The typical presentation of acute pancreatitis includes an elevated serum amylase level and steady, severe upper abdominal pain radiating to the back. ${ }^{3}$ For patients with acute pancreatitis, hospitalization is almost always required. Management includes bowel rest, IV hydration, pain management, supportive care, and serial laboratory tests to monitor for improvement or worsening of the patient's condition. However, not all elevations in amylase are related to pancreatitis.

In 1964, Wilding et al reported a condition found in three patients with persistently elevated serum amylase levels. These patients were found to have amylase-globulin complexes that were too large to be readily excreted by the kidney. In 1967, Berk et al reported a condition in the New England Journal of Medicine which he termed macroamylasemia. Aside from immunoglobulins, the amylase molecule can also bind to polysaccharides forming these enlarged complexes. The size of the complex prohibits renal excretion and results in persistently elevated levels of amylase. Several diseases have been described in association with macroamylasemia, including celiac disease, HIV infection, lymphoma, ulcerative colitis, rheumatoid arthritis, and monoclonal gammopathy. ${ }^{2}$ However, macroamylasemia does not have to be associated with these disease states and can occur in any condition in which abnormal immunoglobulins are present.

Diagnosis of macroamylasemia can be made by applying a simple diagnostic algorithm. ${ }^{3}$ The initial step is to evaluate for elevated serum lipase. If this is also elevated, acute pancreatitis should be strongly considered. The next step is to then measure urinary amylase. If it is elevated, acute pancreatitis should again be strongly considered. If neither of these is the case and renal function is abnormal, secondarily elevated amylase due to renal disease should be considered. Once these causes have been eliminated, isoamylase testing to exclude non-pancreatic sources of amylase can be performed. If the amylase is indeed pancreatic in origin, the amylase-creatinine clearance ratio can be calculated using the formula:

\section{Urine Amylase $\times$ Serum Creatinine $\times 100$ Serum Amylase Urine Creatinine}

If this is found to be less than 1\% macroamylasemia should be suspected. If desired, further analysis can be performed with electrophoresis, polyethylene glycol precipitation testing, and gel chromatography.

Acute pancreatitis needs to be suspected in any patient with abdominal pain and elevated pancreatic enzymes. These 
symptoms, however, need not be related and other causes for elevated pancreatic enzymes should be considered. Nonpancreatic sources of enzymes and other causes for increased release of pancreatic enzymes should be considered. In addition, decreased clearance of enzymes can create elevated serum levels. Decreased clearance can be due to impaired renal function, or abnormal amylase-globulin complexes that can not be readily excreted. An example of the latter is presented here as a case of a patient with abdominal pain and elevated amylase.

\section{References}

1. Vege SS, Chari ST. Clinical manifestations and diagnosis of acute pancreatitis. www.uptodate.com 2007.

2. Gelrud D, Gress FG. Approach to the patient with elevated serum amylase or lipase. www.uptodate.com 2007.

3. LeVine D, Parrish D. Macroamylasemia: A Simple Stepwise Approach to Diagnosis. J Am Bd Fam Pract. 1989; 2:279-282.

4. Wilding P, Cooke WT, Nicholson GI. Globulin bound amylase: cause of persistently elevated levels in serum. Ann Int Med. 1964; 60: 1053-1059.

5. Berk JE, Kizu H, Wilding P, Searcy RL. Macroamylasemia: A Newly Recognized Cause for Elevated Serum Amylase Activity. NEJM. 1967; 277: 941-946. 